\title{
The ATG16L1 risk allele associated with Crohn's disease results in a Rac1-dependent defect in dendritic cell migration that is corrected by thiopurines
}

\author{
ME Wildenberg ${ }^{1,2}$, PJ Koelink ${ }^{1}$, K Diederen $^{1}$, AA te Velde ${ }^{1,2}$, SCS Wolfkamp ${ }^{2}$, VJ Nuij ${ }^{3}$, \\ MP Peppelenbosch ${ }^{3}$, M Nobis ${ }^{4}$, OJ Sansom ${ }^{4}$, KI Anderson ${ }^{4}$, CJ van der Woude ${ }^{3}$, GRAM D’Haens ${ }^{2}$ and \\ GR van den Brink ${ }^{1,2}$
}

Thiopurines are commonly used drugs in the therapy of Crohn's disease, but unfortunately only show a $30 \%$ response rate. The biological basis for the thiopurine response is unclear, thus hampering patient selection prior to treatment. A genetic risk factor associated specifically with Crohn's disease is a variant in ATG16L1 that reduces autophagy. We have previously shown that autophagy is involved in dendritic cell (DC)-T-cell interactions and cytoskeletal regulation. Here we further investigated the role of autophagy in DC cytoskeletal modulation and cellular trafficking. Autophagy-deficient DC displayed loss of filopodia, altered podosome distribution, and increased membrane ruffling, all consistent with increased cellular adhesion. Consequently, autophagy-deficient DC showed reduced migration. The cytoskeletal aberrations were mediated through hyperactivation of Rac1, a known thiopurine target. Indeed thiopurines restored the migratory defects in autophagy-deficient DC. Clinically, the ATG16L1 risk variant associated with increased response to thiopurine treatment in patients with Crohn's disease but not ulcerative colitis. These results suggest that the association between ATG16L1 and Crohn's disease is mediated at least in part through Rac1 hyperactivation and subsequent defective DC migration. As this phenotype can be corrected using thiopurines, ATG16L1 genotyping may be useful in the identification of patients that will benefit most from thiopurine treatment.

\section{INTRODUCTION}

Genome-wide association studies have identified a large number of risk alleles associated with the development of inflammatory bowel disease (IBD). ${ }^{1-3}$ Many of these alleles are shared between Crohn's disease and ulcerative colitis, the two main forms of IBD, ${ }^{4}$ and some with other immune-mediated diseases such as rheumatoid arthritis, systemic lupus erythematosus, and Type 1 diabetes. However, a small number of alleles have been associated specifically with the development of Crohn's disease. This group contains alleles associated with pathogen recognition (e.g., NOD2) and autophagy (e.g., ATG16L1, IRGM, ULK1). Most of these risk alleles are relatively rare, but the ATG16L1 risk allele is homozygous in up to $30 \%$ of patients with Crohn's disease. ${ }^{5}$

While the process of autophagy was already described in the 1970s, it was initially considered a mere energy recycling pathway under nutrient deficient conditions. The association between autophagy and Crohn's disease has resulted in renewed interest in this pathway and its role in inflammation. However, despite many recent publications, the biology underlying the pathogenic role in Crohn's disease remains poorly understood. In the intestinal epithelium, autophagy is involved in the secretory function of Paneth cells, and ATG16L1 deficiency is hypothesized to result in decreased release of

${ }^{1}$ Tytgat Institute for Intestinal and Liver Research, Academic Medical Center, Amsterdam, The Netherlands. ${ }^{2}$ Department of Gastroenterology and Hepatology, Academic Medical Center, Amsterdam, The Netherlands. ${ }^{3}$ Department of Gastroenterology and Hepatology, Erasmus MC, Rotterdam, The Netherlands and ${ }^{4}$ Beatson Institute for Cancer Research, Bearsden, Glasgow, UK. Correspondence: ME Wildenberg or GR van den Brink (m.e.wildenberg@amc.nl or g.r.vandenbrink@amc.uva.nl) 
antimicrobial proteins and subsequent microbial dysbiosis. ${ }^{6,7}$ In addition, ATG16L1 is required to counteract endoplasmic reticulum stress, and thus protect the intestinal epithelium from spontaneous apoptosis. ${ }^{8}$ In innate immune cells, autophagy has been shown to be involved in clearance of intracellular pathogens such as Salmonella enterica and Mycoplasma tuberculosis, as well as in antigen processing. ${ }^{9-11}$ Macrophages lacking ATG16L1 are more susceptible to lipopolysaccharide stimulation and secrete increased amounts of interleukin- $1 \beta .{ }^{12}$ However, the relative contribution of all these mechanisms to the clinical phenotype of Crohn's disease remains unclear.

Crohn's disease is characterized by hyperactive immune responses in the intestine. Dendritic cells (DCs) play a critical role in maintaining immune homeostasis and we have previously shown that autophagy-deficient DC form a hyperstable immunological synapse resulting in increased T-cell activation and Th17 skewing. ${ }^{13}$ These changes were associated with cytoskeletal rearrangements that resulted in an elongated phenotype of the DC. Cytoskeletal rearrangements are at the basis of many biological processes, including cell-cell and cell-matrix interactions and cell motility. Here we further explore the role of autophagy in cytoskeletal organization and find that autophagy regulates cell adhesion and migration through modulation of Racl activity. We then used this data to evaluate the role of ATG16L1 polymorphisms in the clinical response to thiopurines, immunosuppressants that are frequently used in Crohn's disease and have been shown to act as Rac1 inhibitors. ${ }^{14,15}$

\section{RESULTS}

\section{Cytoskeletal changes in autophagy-deficient DC}

The ATG16L1 risk allele results in decreased levels of ATG16L1 protein, and can be mimicked using siRNA knockdown. ${ }^{13}$ Detailed analysis of human monocyte-derived DC (moDC) treated with ATG16L1 siRNA revealed a remarkable change in cytoskeletal organization. These autophagy-deficient moDCs showed significantly reduced numbers of filopodia-like protrusions and a more ruffled phenotype (Figure 1a and Supplementary Figure S1 online). In addition, there was a significant increase in the spread of local adhesion sites known as podosomes (Figure 1b and Supplementary Figure S1). These same cytoskeletal changes were observed after $2 \mathrm{~h}$ treatment with $20 \mu \mathrm{M}$ of the autophagy inhibitor 3-methyladenine (3MA, Figure 1a and Supplementary Movie S1), indicating a role for the process of autophagy rather than a specific function of ATG16L1.

The relative absence of filopodia and enhanced ruffling phenotype of the cell membrane are suggestive of activation of the small Rho GTPase Rac1. ${ }^{16,17}$ To directly test the involvement of Rac1 in the altered phenotype of autophagy-deficient DC, cells were treated with 3MA and the Rac inhibitor EHT1864. Indeed, inhibition of Rac rescued the cytoskeletal defects in the autophagy-deficient DC (Figure 1c).

\section{Autophagy regulates Rac1 activity}

To further confirm the association between Racl and autophagy deficiency, we treated primary human DC with 3MA overnight and analyzed Racl activity using an ELISA-based method (G-LISA). Inhibition of autophagy significantly increased Racl activity both in DC and the monocytic cell line THP-1 (Figure 2a and data not shown). In addition, ATG16L1 knockdown also significantly increased active Rac1 (Figure 2b). To confirm these findings using an independent approach, we assessed Racl activity using Raichu-Racl mice, which carry a Fluorescence Lifetime Imaging with Fluorescence Resonance Energy Transfer (FLIM-FRET) biosensor for Rac1 activity. Bone marrow-derived DC (BMDC) from these animals showed clearly detectable levels of active Rac1. Upon 3MA treatment, Racl activity was increased significantly as indicated by decreased fluorescence lifetime (Figure 2c).Given that autophagy is a degradation pathway, the most likely mechanism underlying the regulation of Racl activity is protein degradation. However, total cellular levels of Rac were not altered significantly by $3 \mathrm{MA}$ treatment in either moDC or THP-1 cells (Figure 2d), contradicting this hypothesis.

\section{Impaired autophagy alters DC migration}

Rac plays an important role in cellular adhesion and migration. In macrophages, Racl and 2 are dispensable for directional migration, but increased Racl activity results in an arrest of macrophage locomotion. ${ }^{18,19}$ It has been proposed that active Rac1 may act to trap macrophages at sites of chronic inflammation, thereby contributing to the perpetuation of the inflammatory response. ${ }^{20}$ We therefore examined autophagydeficient DCs for their adhesive and migratory capacity. Human moDCs treated with ATG16L1 siRNA showed significantly increased adhesion consistent with migration arrest (Supplementary Figure S2). Next, we traced the random migration of 3MA-treated moDCs using time lapse microscopy. Control DCs displayed a non-directional migratory track, covering a median total distance of $270.1 \mu \mathrm{m}$. In the presence of 3MA, the average total distance was reduced significantly to $114.7 \mu \mathrm{m}$ (Figure 3a). Consistent with this, CD11cCre-Atg5 $5^{f l / f l}$ mouse BDMC lacking the autophagy-related protein Atg 5 also travelled significantly less than wild-type controls (112.1 vs. $354.3 \mu \mathrm{m}$, Figure $3 \mathbf{b}$ ). To test the role of autophagy in directional migration, labeled THP1 monocytic cells were added to the top compartment of a transwell filter and migration was induced by the chemokine CCL2. Directional migration of THP1 cells was significantly impaired in the presence of 3MA despite expression of similar levels of various adhesion molecules and chemokine receptors (Figure $3 \mathrm{c}$ and Supplementary Figure S3). Consistently, $\operatorname{Atg} 5^{\text {fl/fl}}$ mutant mouse BMDC showed the same migration defect (Figure 3d). As ATG16L1 siRNA may not result in uniform knock down in all DC we hypothesized that cells with inefficient knockdown would migrate better than cells in which knockdown was more successful. To test this, we applied ATG16L1 siRNA-treated human moDCs to a transwell filter and used quantitative RT-PCR to measure the amount of ATG16L1 relative to glyceraldehyde 3-phosphate dehydrogenase in both compartments after $2 \mathrm{~h}$. Cells that migrated to the bottom compartment expressed more ATG16L1, providing further evidence for a role of ATG16L1 in the migration of DC (Figure 3e). Finally, we tested the role of autophagy in DC 
a
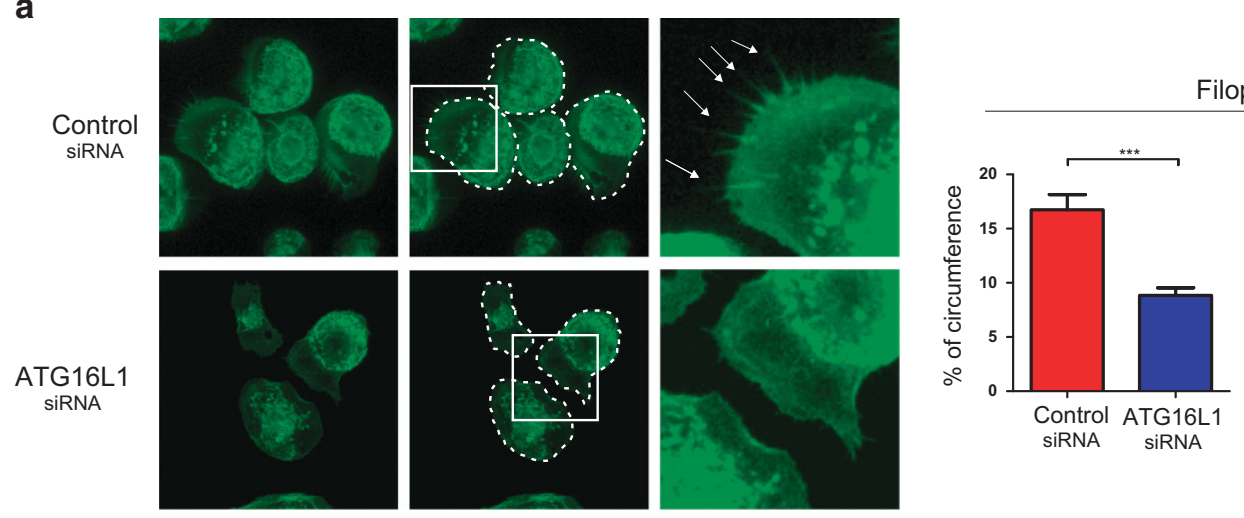

Filopodia

b
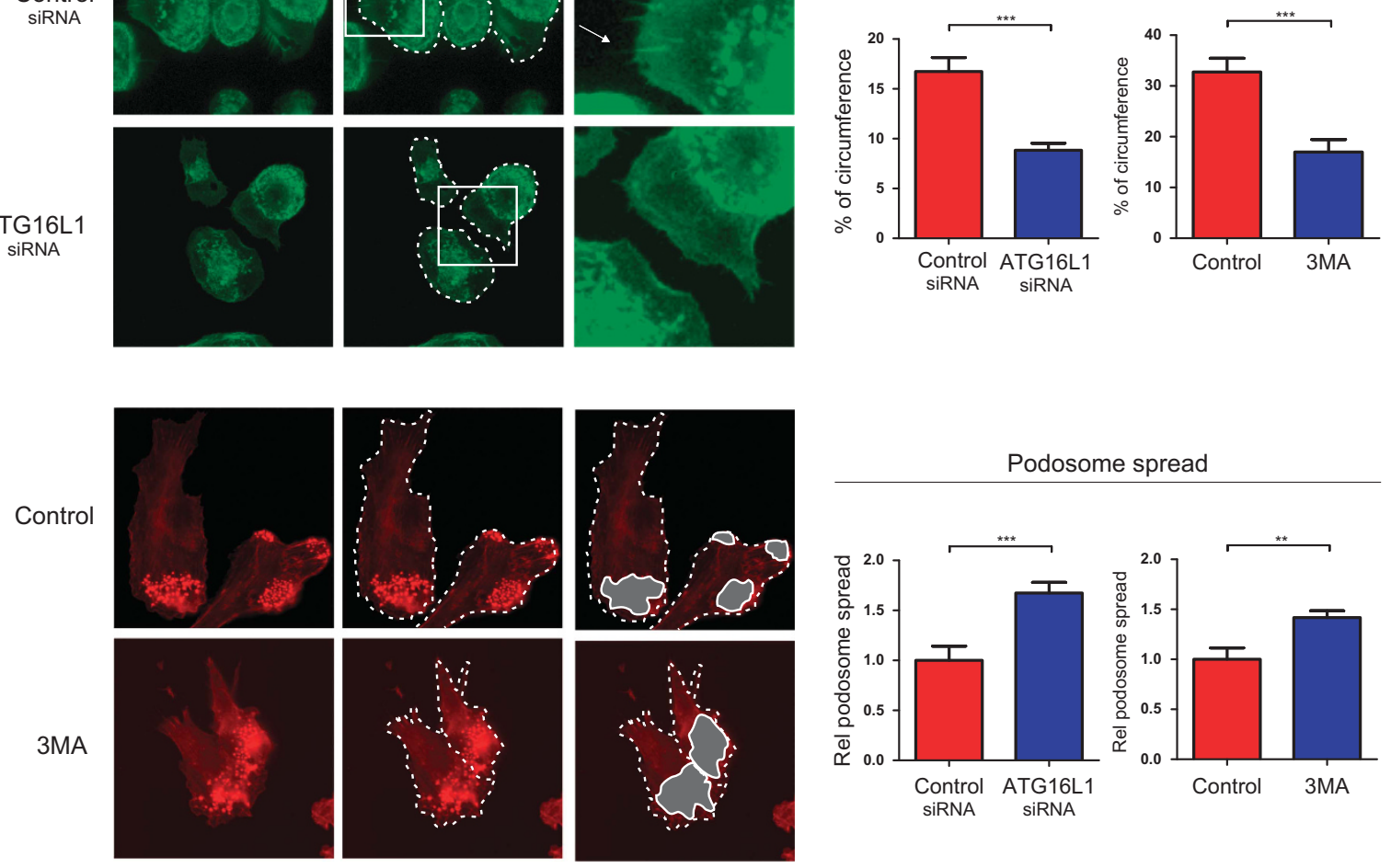

C
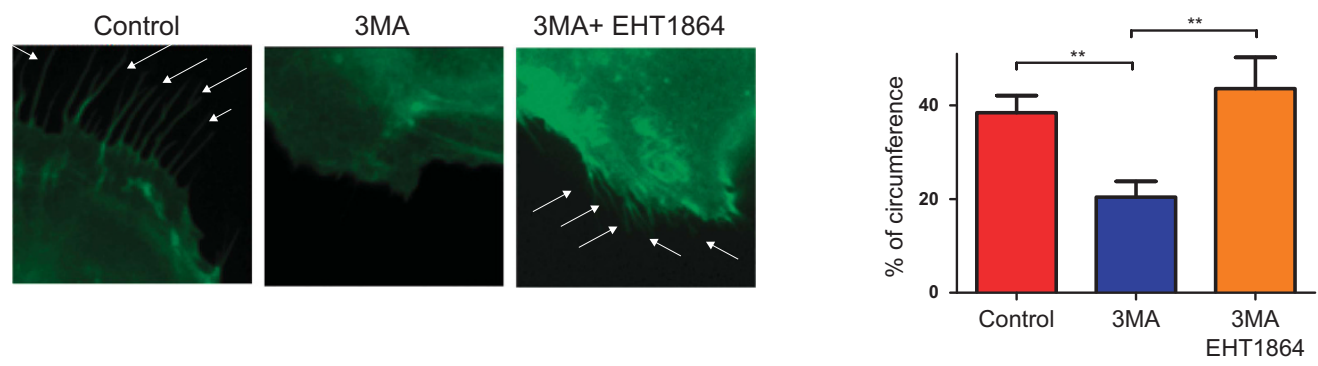

Figure 1 Decreased autophagy results in altered DC morphology. (a) F-actin visualized by phalloidin staining (green). Images show representative moDC after treatment with either control or ATG16L1 siRNA. Middle panel indicates cell borders (dotted lines) and area of magnification (solid lines). The fraction of cell circumference containing visible filopodia was analyzed in three separate experiments in individual donors, representative experiment is shown (left $n=52 /$ sample, right control $n=120$, ATG16L1 $n=107$, Mann Whitney Utest). (b) F-actin visualized by phalloidin staining (red). Images show representative moDC after treatment with either control or 3MA. Middle panel indicates cell borders (dotted lines) and right panel indicates areas as marked for podosome spread (solid lines). The relative spread of podosomes was calculated as the fraction of cell area covered by podosome patches relative to the average of control. Three separate experiments were performed using individual donors, representative experiment is shown (left control $n=19$, 3MA $n=21$, right control $n=23, A T G 16 L 1 n=39$, Mann Whitney Utest). (c) F-actin staining in moDC after treatment with 3MA and Rac1 inhibitor EHT1864 $(20 \mu \mathrm{M})$ for $2 \mathrm{~h}$. The fraction of cell circumference containing filopodia was analyzed in two individual donors, a representative experiment is shown (Control $n=18$, 3MA $n=27$, EHT $n=11$, Kruskall-Wallis test with Dunn's post-hoc analysis). All bars represent mean and s.e.m. ${ }^{\star *} P<0.01$, ${ }^{* * *} P<0.001$. DC, dendritic cell; moDC, monocyte-derived DC.

migration in vivo. $1.5 \times 10^{5}$ fluorescently labeled Atg 5 knock out (KO) DCs were injected in the foot of wild-type mice and an equal number of labeled wild-type DC was injected in the contralateral foot. The number of Atg 5 mutant DC that arrived in the popliteal lymph node after $24 \mathrm{~h}$ was reduced 2.4-fold compared to wild-type DC (Figure 3f).

Thiopurines restore autophagy-mediated migratory defects Thiopurines are commonly used drugs in IBD and their active metabolite 6-thioguanin (6-TG) has been shown to inhibit Rac1 activity by destabilization of the Rac1-Vav complex. ${ }^{14,15} \mathrm{We}$ therefore analyzed whether 6-TG could correct the hyperactivation of Racl and migration defects observed in DC with reduced autophagy. 6-thioguanin restored the migratory capacity of THP1 cells after treatment with 3MA (Figure 4a). Similarly, the migratory defects seen in mouse BMDC lacking Atg5 were reversed by addition of 6-TG (Figure $4 \mathbf{b}$ ). To test the validity of these findings in the context of the physiologically relevant single nucleotide polymorphism (SNP), we isolated monocytes from human 
b Control
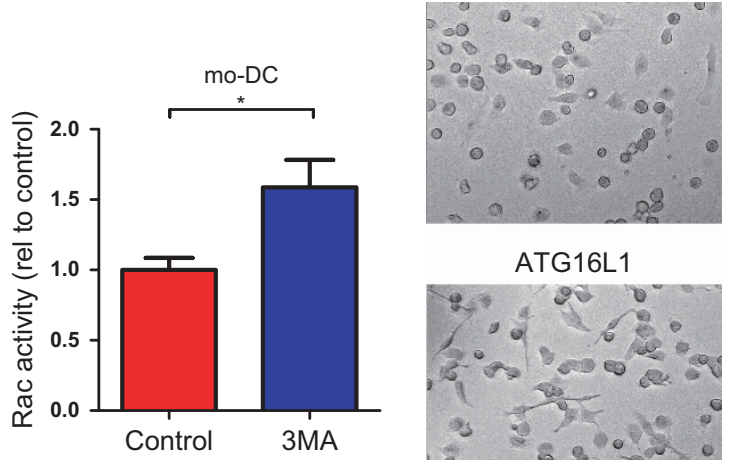

ATG16L1
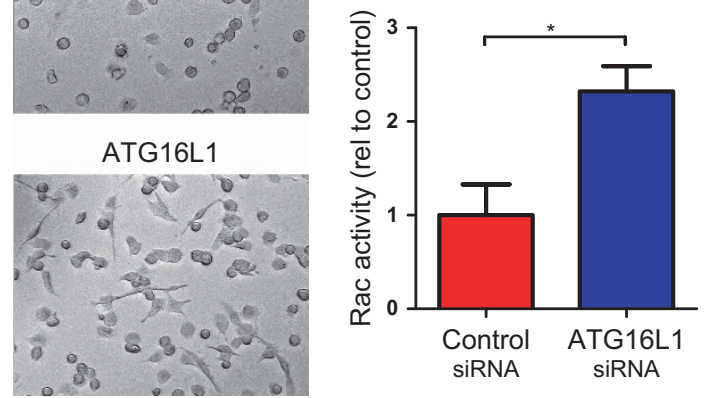

C
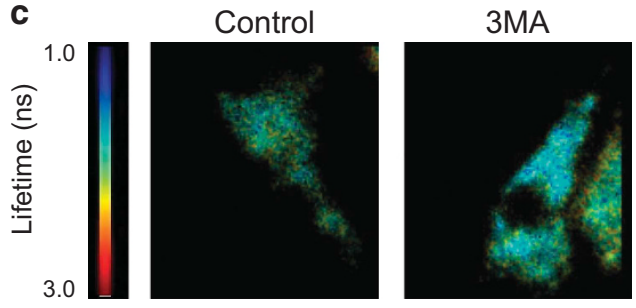

d

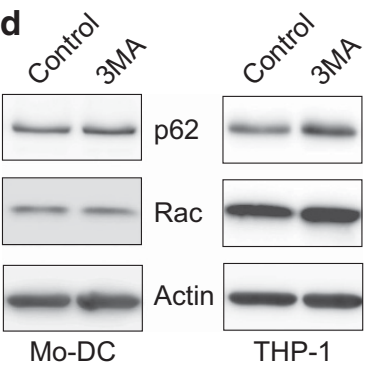

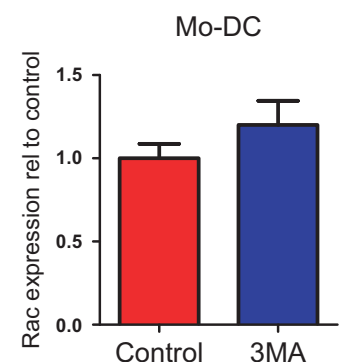

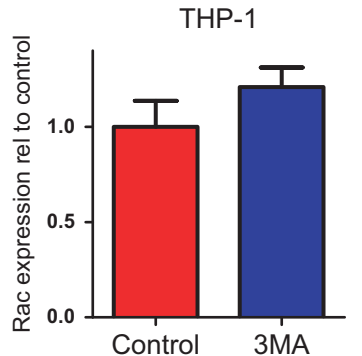

Figure 2 Decreased autophagy results in increased Rac1 activity. (a) Rac1 activity as measured by G-LISA in the presence or absence of overnight treatment with 3MA, Mann Whitney $U$ test. (b) Rac1 activity in moDC after treatment with siRNA. Representative image is shown, data represent three separate samples, all measured in duplicate, Mann Whitney $U$ test. (c) Rac1 activity as measured by FRET-FLIM in the presence or absence of 3MA overnight (control $n=342$, 3MA $n=304$, Mann Whitney $U$ test). (e) Protein levels determined by Western blot in moDC or THP1 cells after overnight incubation with $20 \mu \mathrm{m} 3 \mathrm{MA}$. Both blots representative of three experiments. Graphs depict band intensity corrected for actin and relative to the average of control. All bars represent mean and s.e.m. ${ }^{*} P<0.05$, ${ }^{* * \star} P<0.001$. 3MA, 3-methyladenine; FLIM-FRET, Fluorescence Lifetime Imaging with Fluorescence Resonance Energy Transfer; moDC, monocyte-derived dendritic cell.

donors either wild type or homozygous for the T300A risk allele of ATG16L1. In line with our previous data, DC generated from a risk allele carrier displayed decreased migration compared to wild-type DC (Figure 4c). In addition, DC generated from risk allele carriers displayed a significantly higher level of Rac1 activation (Figure 4d). Treatment with 6-TG reduced Rac1 activity in the risk allele carriers back to wild-type levels, while activity in wild-type cells was not altered. Moreover, 6-TG also rescued the migratory effect seen in T300A risk allele carriers, but did not affect migration in wild types (Figure 4e). Together, these data suggest that the migration defect seen in autophagy-low DC is mediated by Rac1 hyper-activation, and that this defect is selectively corrected by thiopurines.

\section{Thiopurine response correlates with ATG16L1 genotype in Crohn's disease patients}

Prospective randomized studies have shown that only a minority of patients fully responds to thiopurine monotherapy. Complete corticosteroid-free clinical remission is around 30\% after 26 weeks of treatment in patients with Crohn's disease. ${ }^{21}$ Our data suggest that the ATG16L1 polymorphism associated with the development of Crohn's disease results in abnormal cell function due to Rac1 hyper-activation. Given the capacity of thiopurines to inhibit Racl activity, the increased Rac1 activity under autophagy-deficient conditions and the selective effect of 6-TG observed on cells homozygous for the T300A allele, we hypothesized that thiopurines may be particularly effective in patients with Crohn's disease that are homozygous 
a

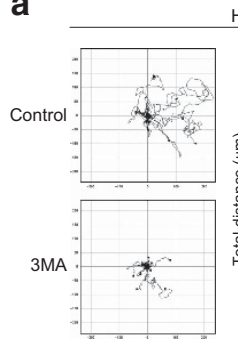

d

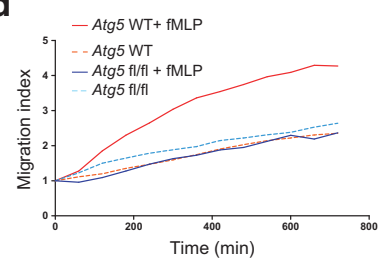

Human

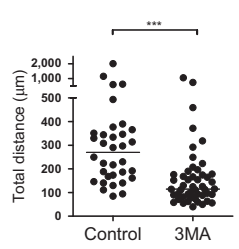

b

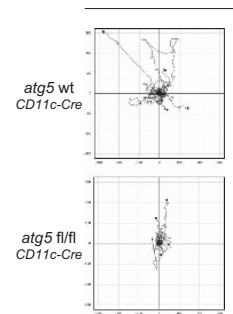

Mouse

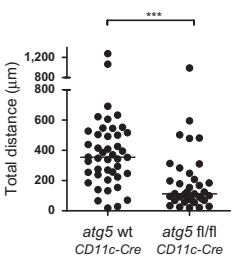

e
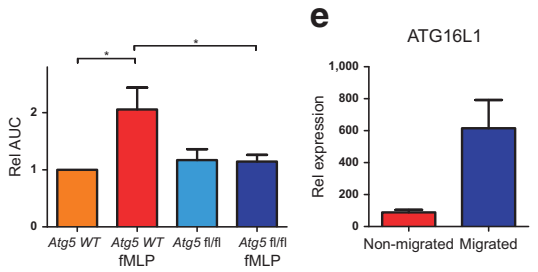

C
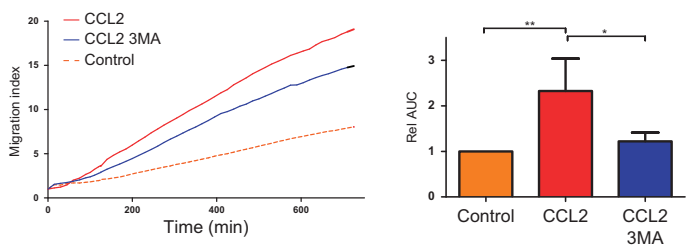

f

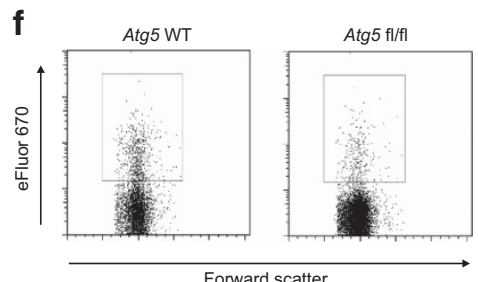

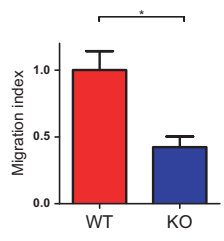

Figure 3 Autophagy knockdown results in decreased migration. (a,b) Trajectories of moDC (human) or BMDC (mouse) in the absence of chemotactic stimuli. Each line represents an individual cell. Graph shows summarized data of two experiments in two separate donors (human) or duplicate samples (mouse). Dots in graph represent individual cells, line indicates median (human control $n=34,3 \mathrm{MA} n=50$, mouse WT $n=44, \mathrm{KO} n=38$, Mann Whitney $U$ test). (c,d) Chemotactic migration of THP-1 cells and BMDC in a transwell system over time. Chemotactic stimuli were CCL2 (20 ng/ml, THP1) and fMLP $(10 \mu \mathrm{M}, \mathrm{BMDC})$. Graphs show representative experiments, bars show area under the curve analysis of summarized data $(n=7$ individual experiments, Kruskall-Wallis test). (e) MoDCs were allowed to migrate for $1 \mathrm{~h}$ through a transwell insert in response to $10 \mu \mathrm{m} f M L P$. Migrated and nonmigrated cells were harvested from the bottom and top of the filter respectively. ATG16L1 expression was determined by quantitative RT-PCR. Graph shows representative data of experiments in three individual donors, $n=3$. (f) Migration of BMDC in vivo. Cultured BMDC were labeled with eFluor670 and injected into the foot of WT animals $(1.5 \times 10 \mathrm{E} 6 \mathrm{cells} / \mathrm{foot})$. Popliteal lymph nodes were analyzed for the presence of tracker + cells $24 \mathrm{~h}$ later. Data from two separate experiments are shown, WT $n=6, \mathrm{KO} n=5$, Mann Whitney $U$ test. All bars represent mean and s.e.m. ${ }^{\star} P<0.05$, ${ }^{* \star} P<0.01$,

${ }^{* * *} P<0.001$. 3MA, 3-methyladenine; BMDC, bone marrow-derived dendritic cell; KO, knock out; moDC, monocyte-derived dendritic cell; WT, wild type.

carriers of the ATG16L1 risk allele. To test this hypothesis, we determined the response to thiopurines (for definition of response see methods) in patients that were genotyped for ATG16L1 in our well-characterized IBD cohort at the AMC in Amsterdam (Table 1).

In Crohn's disease, patients homozygous for the T300A risk allele were significantly more likely to respond to thiopurine therapy than patients carrying at least one wild-type allele (response rates 81 vs. $60 \%$ respectively, odds ratio $2.8, P<0.05$ ). In contrast, in ulcerative colitis, no correlation between ATG16L1 genotype and thiopurine response was found (odds ratio $1, P=0.94$ ). This is in line with the observation that the ATG16L1 SNP is not associated with development of disease in ulcerative colitis. The ATG16L1 polymorphism-associated phenotype is therefore most likely not part of the pathology in these patients, and correction of the phenotype does not alter disease course. We further analyzed this in a second independent cohort of IBD patients from the Erasmus MC in Rotterdam. In this cohort, homozygosity for T300A was also associated with a higher thiopurine response rate in Crohn's disease ( 81 vs. $53 \%$, odds ratio $3.8, P<0.001$ ). Again, in ulcerative colitis, no correlation was observed (odds ratio 1.0, $P=0.93$ ).

\section{DISCUSSION}

SNPs in genes involved in the regulation of autophagy are selectively associated with the risk of developing small intestinal and colonic Crohn's disease. A role for autophagy has been described in various biological mechanisms that may be related to the development of Crohn's disease. These mechanisms include Paneth cell function, intracellular microbial handling, regulation of endoplasmatic reticulum stress, and interleukin$1 \beta$ secretion. ${ }^{6-12}$ The extent to which each of these mechanisms contribute to the risk of developing Crohn's disease and possibly disease activity and progression remains to be elucidated.

We have previously found that autophagy in DC plays a key role in the regulation of the stability of the immunological synapse that forms between DC and T cells during cognate antigen recognition. ${ }^{13}$ Here we find that suppression of autophagy in DC results in Rac1 hyperactivation and subsequent migration defects. Defective immune cell migration has already been linked to the development of IBD. For example, patients with Wiskott Aldrich Syndrome carry a mutation in Wiskott Aldrich Syndrome protein (WASp), a cytoskeletal regulator, resulting in severely impaired migration of immune cells. $^{22}$ Besides their immunodeficiency, these patients are characterized by a strongly increased risk for immunemediated diseases, including IBD. In mice, it has been shown that the colitis is caused by WASp deficiency in innate immune cells and not in $\mathrm{T}$ or $\mathrm{B}$ cells. $^{23}$

The altered cytoskeletal phenotype in autophagy-low DC was reminiscent of Rac1 overactivation, ${ }^{16}$ and we indeed found increased Rac1 activity in both 3MA and ATG16L1 siRNAtreated DC. In line with this, an earlier study described decreased Rac1 activity as a result of starvation-induced autophagy. ${ }^{24}$ In both the previous study and ours, modulation of autophagy did not alter total Racl protein levels and the underlying mechanism of autophagy-mediated Racl regulation 
a

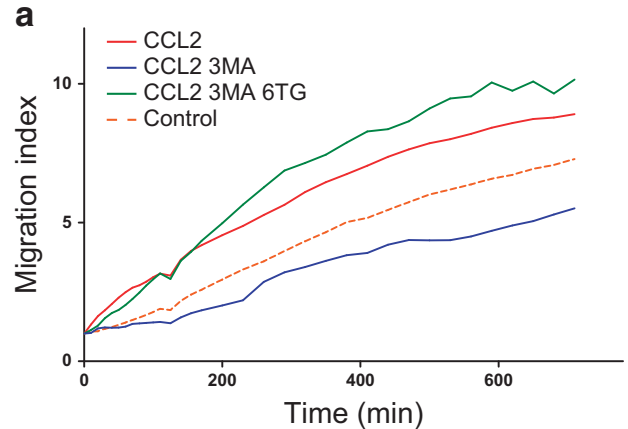

b

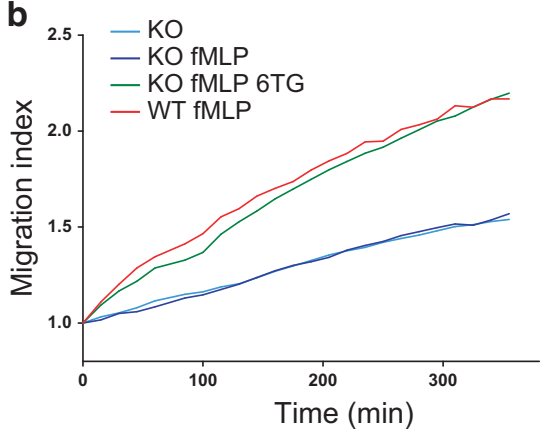

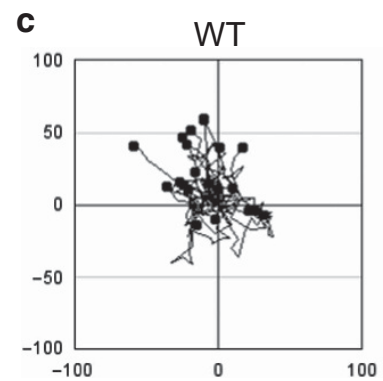
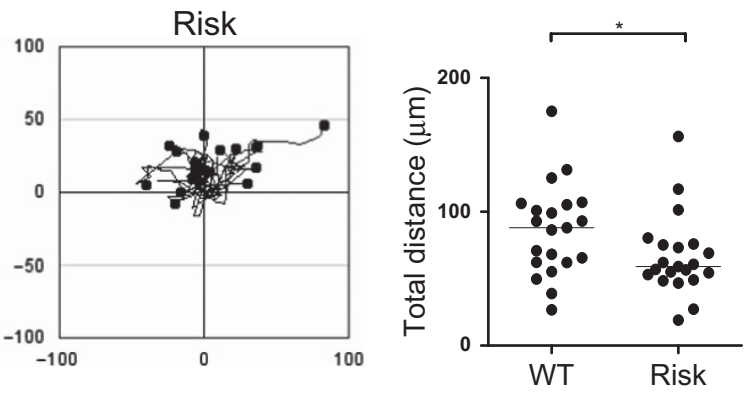

d

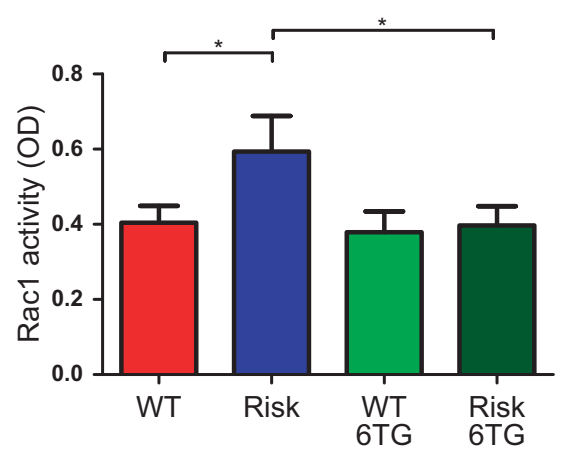

e

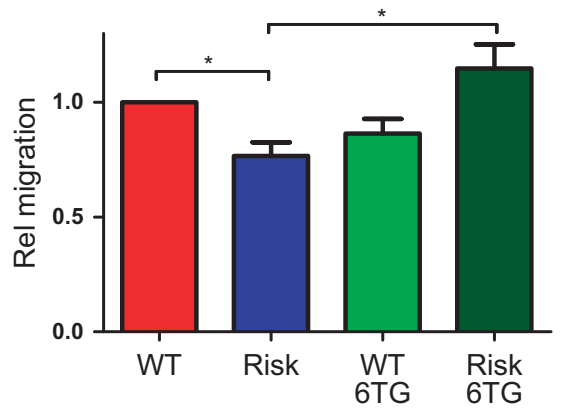

Figure 4 Thiopurine treatment reduces Rac1 hyperactivation and restores migratory capacity in autophagy-impaired DC. (a,b) Chemotactic migration of THP-1 cells and BMDC in a transwell system over time. Graphs show representative figure of five (a) or four (b) individual experiments. (c) Trajectories of moDC. Each line represents an individual cell. Dots represent individual cells, line indicates median (both $n=21$, Mann Whitney $U$ test). (d) Rac activity in moDC as measured by G-LISA after overnight treatment with 6TG (all bars $n=3$ donors, Friedman test for donor paired samples). (e) Random migration of human monocytes derived from ATG16L1 rs2241880 wild-type (A allele) or homozygous risk ( $G$ allele) carriers. Monocytes were plated and images by time lapse microscopy every $5 \mathrm{~min}$. Average velocity was determined over a $2-4 \mathrm{~h}$ period using the manual tracker plugin of Image $\mathrm{J}$. Graph represents four individual experiments in eight separate donors (1 WT, 1 risk per experiment). Kruskall-Wallis test. All bars represent mean and s.e.m. ${ }^{\star} P<0.05$. DC, dendritic cell; KO, knock out; moDC, monocyte-derived DC; WT, wild type.

remains to be elucidated. One possibility is the specific autophagic degradation of active Rac. Previous work has shown that upon activation, Racl is ubiquitinated and subsequently degraded. ${ }^{25}$ Although this was presumed to be proteosomally mediated, ubiquitination has now been shown to target proteins for both proteosomal and autophagosomal degradation. ${ }^{26}$ As this would occur only at local sites of Rac1 activation (i.e., adhesion sites), this may not be detectable in the much larger total cell pool of Rac1 while still having profound functional effects. ${ }^{27}$ A second option may be that autophagy modulates the level of the Racl modulating GTPase activating proteins (GAPs) and guanine nucleotide exchange factors (GEFs) and thereby has a secondary effect on Racl activity. Finally, the increased Racl activity may be a compensatory mechanism counteracting decreased Rac2 activity. All three options, which are not mutually exclusive, are topics of current investigation.

A recent study described the increased susceptibility to the development of colitis in CD11cCre-Atg5 ${ }^{\text {fl/fl }}$ and CD11cCreAtg fllfl $^{f}$ animals, both of which lack autophagy in DC. ${ }^{28}$ However, the functional mechanisms underlying this observation were not explored. Our data suggest that the phenotype of these mice may at least partially be related to defective DC migration. There are several ways in which defective DC migration may contribute to the risk of developing IBD. First, myeloid cell egress is an important part of the resolution and clearing of inflammatory infiltrates in peripheral tissues. In two animal models of IBD, stimulating the number of myeloid cells egressing from the lymph nodes resulted in decreased disease severity. ${ }^{29}$ Second, induction of oral tolerance is mediated by 
Table 1 Correlation between ATG16L1 rs2241880 and thiopurine response in IBD patients

\begin{tabular}{llrcrrr}
\hline & Genotype & $\boldsymbol{n}$ & $\begin{array}{c}\text { Responder } \\
\boldsymbol{n}(\%)\end{array}$ & $\begin{array}{c}\text { Non-responder } \\
\boldsymbol{n}(\%)\end{array}$ & OR & P-value \\
\hline $\begin{array}{l}\text { Cohort 1 } \\
\text { All patient }\end{array}$ & & 283 & & & & \\
CD & WT/Het & 137 & $82(60)$ & $55(40)$ & & \\
& Risk & 31 & $25(81)$ & $6(19)$ & 2.79 & 0.03 \\
UC & WT/Het & 88 & $58(66)$ & $30(34)$ & & \\
& Risk & 27 & $18(67)$ & $9(33)$ & 1.03 & 0.942
\end{tabular}

Cohort 2

\begin{tabular}{llrrrrr} 
All patient & \multicolumn{7}{c}{330} & & & & & \\
CD & WT/Het & 160 & $85(53)$ & $75(47)$ & & \\
& Risk & 84 & $68(81)$ & $16(19)$ & 3.75 & $<0.001$ \\
UC & WT/Het & 60 & $41(69)$ & $19(31)$ & & \\
& Risk & 26 & $18(69)$ & $8(31)$ & 1.04 & 0.934 \\
\hline
\end{tabular}

Abbreviations: IBD, inflammatory bowel disease; $n$, number; OR, odds ratio; WT, wild type.

Genotype for rs2241880 (wild type A, risk allele G), use of and response to thiopurines was determined in two independent cohorts of IBD patients (for definitions of response, see Materials and Methods). Distributions were analyzed using Pearson's Chi square test. OR: odds ratio of response compared to patients carrying at least one wild-type allele.

intestinal DC, which sample luminal antigen and subsequently migrate to the draining mesenteric lymph nodes. ${ }^{30}$ Mice lacking the chemokine CCR7 have normal numbers of DC in the lamina propria, but their DC fail to migrate to the mesenteric lymph node and induction of oral tolerance is severely hampered in these animals. ${ }^{31}$ Together with the increased T-cell activation and Th17 skewing induced by these autophagy-deficient $\mathrm{DC},{ }^{13}$ these mechanisms may contribute to the development of non-resolving chronic inflammation.

Thiopurines are commonly used drugs in IBD that all have in common the generation of the metabolite 6-TG. Although this compound was initially generated as a DNA synthesis inhibitor, it has now clearly been shown that it also has inhibitory effects on the GTPase Rac1 by destabilizing the Rac1-Vav activation complex. In particular in the relatively low dosing used in IBD, this Rac1 inhibitory effect is likely to be the main mechanism of action. ${ }^{14,15}$ However, these previous studies focused on the effect of Rac1 inhibition in T cells, despite the fact that increased levels of Rac1 have been described in colonic myeloid cells in Crohn's disease. ${ }^{32}$ Additionally, the reason why Racl activity was increased was not clear from these studies. We now describe that autophagy deficiency caused by the ATG16L1 SNP results in increased Rac1 activity and subsequent functional defects in migration in DC. In vitro, both Racl activity and the migratory defect could be corrected using thiopurines. Strikingly, thiopurines only affected DC migration in autophagy-deficient cells. Basal levels of Rac1 activity as seen in DC obtained from wild-type ATG16L1 donors were not altered by 6-TG, and migration was undisturbed. This suggests that thiopurines would be particularly effective in $\mathrm{CD}$ patients with increased Rac1 activity, i.e., those carrying the ATG16L1 T300A risk allele. In a retrospective study, we indeed found a correlation between ATG16L1 genotype and response to thiopurines, with patients homozygously carrying the risk allele having increased chances of responding. In ulcerative colitis, ATG16L1 is not associated with development of disease. In line with this, the ATG16L1 genotype did not have any effect on the response to thiopurine treatment in this patients group. The frequency of the risk allele in cohort 1 was somewhat lower than previously reported, probably due to the lower number of patients compared to the genome-wide association studies. However, the thiopurine response in relation to the ATG16L1 genotype was consistent between this cohort and the replication cohort, which did show expected frequencies of the T300A risk allele. These data suggest that the effector mechanism of thiopurines in ulcerative colitis differs from that in Crohn's disease.

Since only around $30 \%$ of patients with Crohn's disease treated with the thiopurine azathioprine are in complete steroid-free clinical remission after six months of therapy, ${ }^{21}$ and thiopurines can have considerable side effects, our observation that the ATG16L1 risk allele associates with the response to thiopurines may help to select patients most likely to benefit from this therapy. However, although thiopurine response was better in patients homozygously carrying the ATG16L1 risk allele, a proportion of patients carrying the wild-type allele also respond to this therapy. In these patients, thiopurine effects may either be mediated through an additional, non-Racl dependent, mechanism, or Rac1 may indeed be dysregulated but due to other (genetic) factors.

In summary, our data link polymorphisms in ATG16L1 to DC migration defects via increased Rac1 activity. These defects can be corrected using thiopurine therapy. Our findings may help to identify patients more likely to respond to thiopurines in Crohn's disease and suggest new pathways for therapeutic intervention.

\section{MATERIALS AND METHODS}

Mice. CD11c-eGFP-Cre mice were obtained from Jackson Laboratories (Bar Harbour, ME). Atg5 ${ }^{\text {lox }}$ animals have been described previously ${ }^{33}$ and were provided by Prof N. Mizushima (University of Tokyo, Tokyo, Japan). CD11c-eGFP-Cre-Atg5 mice were bred in the animal facility of the AMC and supplied with water and chow ad libitum. For comparison between Atg5 $\mathrm{KO}$ and WT BMDC, bone marrow was obtained from litter mates at the age of 8-20 weeks. Wild-type female C56Bl/6 animals were obtained from Harlan and included between the ages of 8-20 weeks. Rac-FRET mice expressing the Raichu-Rac biosensor have been described previously. ${ }^{34}$ All experimental procedures were approved by the local Animal Ethics Committee in accordance with national guidelines.

For in vivo migration, BMDC were cultured as described. On days 7-9 of culture, cells were harvested and labeled using eFluor670 (eBioscience, San Diego, CA). Cells were then injected subcutaneously on the top of the foot of the hind leg $(1.5 \times 105$ cells/injection $)$. After $24 \mathrm{~h}$, popliteal lymph nodes were harvested and analyzed by flow cytometry. Migration index was calculated as $\%$ of label + cells relative to the average of control.

Cell isolation and culture. For generation of human DC, monocytes were isolated from healthy donor buffy coats by percoll gradient centrifugation and cultured for 6-8 days in AIM-V culture medium (Invitrogen, Carlsbad, CA) in the presence of recombinant human granulocyte macrophage colony stimulating factor and interleukin-4 
(both $50 \mathrm{ng} / \mathrm{ml}$, RnD Systems Minneapolis, MN). For knockdown studies, ON-TargetPlus ATG16L1 specific as well as control siRNA pools were obtained from Dharmacon (Epsom, UK). Both were transfected using Dharmafect 4 (Dharmacon) transfection reagent according to the manufacturer's protocol. In all cases negative control siRNA was used as the control sample to correct for nonspecific siRNA effects.

For generation of mouse BMDCs, bone marrow was isolated from murine femur and cultured in RMPI 1640 culture medium (Invitrogen), in the presence of $10 \%$ fetal calf serum and $20 \mathrm{ng} / \mathrm{ml}$ recombinant mouse granulocyte macrophage colony stimulating factor (RnD Systems) for 7-9 days. THP1 cultures were tested and found negative for mycoplasma contamination.

Immunofluorescence. F-actin was visualized using fluorescently labeled phalloidin (Invitrogen). Cells were adhered to glass coverslips, fixed in $4 \%$ paraformaldehyde, stained in permeabilization buffer (phosphate buffered saline containing $0.05 \%$ Triton X-100), and embedded in SlowFade Gold (Invitrogen). Cells were imaged using a Leica DM6000 Digital Microscope equipped with LAS AF Software (Leica, Wetzlar, Germany) or an SP-8 X Confocal microscope (Leica). Image processing and quantification was performed using ImageJ software (http://imagej.nih.gov/ij/).

FLIM-FRET imaging. Fluorescence lifetime microscopy measurements were conducted using a Lambert Instruments LIFA system fluorescence attachment on a Nikon Eclipse TE 2000-U microscope equipped with a $\times 60$ objective and a filter block consisting of a 436/20 excitation filter, a T455LP dichroic mirror, and a 480/40 emission filter. FLIM-FRET was measured by frequency domain as described previously. ${ }^{35}$ Briefly, a modulated $445 \mathrm{~nm}$ LED was used as light source and fluorescein $(10 \mu \mathrm{M}$ in $0.1 \mathrm{M}$ Tris- $\mathrm{Cl}, \mathrm{pH}>10)$ as reference standard with a known lifetime of $4.0 \mathrm{~ns}$. The donor lifetime (ECFP), $\tau$, was analyzed using the FLIM software (version 1.2.12; Lambert Instruments, the Netherlands). Representative images of three independent experiments are shown.

Protein analysis. For Western blot analysis, cells were lysed in commercial lysisbuffer (Cell Signaling, Beverly, MA) containing Protease Inhibitor Cocktail (MP Biomedicals, Solon, OH), and homogenized by ultrasound sonication. Samples were run on sodium dodecyl sulfate polyacrylamide gel electrophoresis gels under reducing conditions and transferred to a Protran BA85 filter (GE Health Care, Zeist, the Netherlands). Membranes were blocked by incubation in $5 \%$ non-fat milk, (Nutricia, Wageningen, the Netherlands). Antibodies used for detection were anti-Rac1/2/3 (\#2465, Cell Signaling), anti-p62 (clone 3/P62, BD Biosciences, San Jose, CA), and anti-beta actin (Sigma, Deisenhofen, Germany). Expression was detected by Lumilight Plus (Roche, Woerden, the Netherlands).

Migration assays. For random migration, cells were plated in culture plates and maintained at $37^{\circ} \mathrm{C}$ and $5 \% \mathrm{CO}_{2}$ during imaging. Time lapse imaging was performed using a Leica IRBE inverted microscope fitted with a culture chamber or a Cytomate cell imager (Cytomate technologies, Eindhoven, the Netherlands) placed in a standard incubator. Cell tracking and analysis was performed using ImageJ software.

For chemokine-induced migration, cells were labeled by incubation in $10 \mu \mathrm{M}$ CFSE (Invitrogen), washed and resuspended in RPMI 1640 culture medium without phenol red (Lonza, Verviers, Belgium). Cells were then placed in the upper chamber of a Fluoroblok transwell insert (BD Biosciences). A chemotactic agent (fMLP $10 \mu \mathrm{M}$, Sigma or recombinant human CCL2 $20 \mathrm{ng} / \mathrm{ml}$, Peprotech, Rocky Hill, NJ) was placed in the lower chamber and plates were incubated at $37^{\circ} \mathrm{C}$. Fluorescent signals from both the upper and lower chambers were measured every $15 \mathrm{~min}$. Migration index was calculated as Fluorescence in lower chamber/Fluorescence in upper chamber, with the index at the start of migration set to 1 . For statistical purposes, data are represented as area under the curve of individual samples.
Quantitative RT-PCR analysis. RNA was isolated using the RNAeasy mini kit (Qiagen, Hilden, Germany) and cDNA was generated using RevertAid reverse transcriptase (Thermo Scientific, Waltham, MA) and random primers (Promega, Leiden, the Netherlands). Quantitative RT-PCR reactions were carried out using Quantitekt primer assays and SybrGreen PCR Kit (both Qiagen). For relative expression, all data were normalized against expression of the household gene glyceraldehyde 3-phosphate dehydrogenase.

Adhesion assays. For cell adhesion assays, cell suspensions were added to tissue culture plates that were either uncoated or coated with fibronectin (Sanquin, Amsterdam, the Netherlands) and incubated for times indicated at $37^{\circ} \mathrm{C}$. Afterwards, plates were shaken at $2000 \mathrm{rpm}$ for $15 \mathrm{~s}$ and non-adherent cells were washed away. Adherent cells were fixed in $4 \%$ paraformaldehyde and stained using Crystal Violet (Sigma, $5 \mathrm{mg} / \mathrm{ml}$ in $2 \%$ ethanol). After washing and drying, Crystal Violet was solubilized using 1\% SDS and measured at $550 \mathrm{~nm}$.

Patient studies. To determine a potential association between thiopurine use and ATG16L1 genotype we performed a cross-sectional analysis of two independent cohorts. We included patients treated for IBD at the Academic Medical Center in Amsterdam, the Netherlands (cohort 1) or the Erasmus MC in Rotterdam, the Netherlands (cohort 2) that had previously been genotyped for ATG16L1 risk allele rs2241880. ${ }^{36,37}$ The medical records of these patients were analyzed by an investigator that was blinded for the patient genotype and assessed for exposure to thiopurines. Only patients with a history of thiopurine use (Azathioprine, 6-mercaptopurine or 6-TG) were further assessed. We excluded patients who either stopped thiopurine use because of intolerance or were immediately started on anti-TNF/thiopurine combination therapy and had never received thiopurine monotherapy from the analysis of this study. Response to thiopurines was defined as continued use up to the moment of assessment or cessation because of prolonged disease remission on thiopurine monotherapy. Nonresponse was defined as cessation of therapy because of a recorded lack of response, or the need to add steroids or start treatment with antiTNF antibodies. Cohort characteristics are shown in Supplementary Table 1.

Statistics. Statistical analyses were performed using GraphPad Prism (Graphpad software, La Jolla, CA) or SPSS (SPSS, Chicago, IL) software. For two-group analysis, Mann Whitney $U$ test (unpaired samples) or Wilcoxon-signed-rank test (paired samples) was used, for multiple groups Kruskall-Wallis followed by Dunn's post-hoc analysis. For the retrospective cohort studies, distribution among categories was analyzed using Pearson's Chi-Square test. All tests were performed two-sided. Data were considered significant when $P<0.05$.

SUPPLEMENTARY MATERIAL is linked to the online version of the paper at http://www.nature.com/mi

\section{ACKNOWLEDGMENTS}

Atg5 $5^{\text {lox }}$ animals were provided by Prof N. Mizushima (University of Tokyo, Tokyo, Japan).

\section{AUTHOR CONTRIBUTION}

M.E.W., P.J.K., and M.N. performed experiments and analyzed and discussed data, K.D. collected and analyzed clinical data, A.A.t.V., C.J.v.d.W., V.J.N., and S.C.W. collected clinical samples and data and analyzed clinical data, M.P.P., K.I.A., and O.J.S. discussed data and strategy, G.R.v.d.B. and G.R.d.H. supervised the study and M.E.W. and G.R.v.d.B. wrote the manuscript with input of all authors.

\section{DISCLOSURE}

The authors declare no conflicts of interest.

c) 2017 Society for Mucosal Immunology 


\section{REFERENCES}

1. Barrett, J.C. et al. Genome-wide association defines more than 30 distinct susceptibility loci for Crohn's disease. Nat. Genet. 40, 955-962 (2008).

2. Franke, A. et al. Genome-wide meta-analysis increases to 71 the number of confirmed Crohn's disease susceptibility loci. Nat. Genet. 42, 1118-1125 (2010).

3. McGovern, D.P. et al. Genome-wide association identifies multiple ulcerative colitis susceptibility loci. Nat. Genet. 42, 332-337 (2010).

4. Lees, C.W., Barrett, J.C., Parkes, M. \& Satsangi, J. New IBD genetics: common pathways with other diseases. Gut 60, 1739-1753 (2011).

5. Hampe, J. et al. A genome-wide association scan of nonsynonymous snps identifies a susceptibility variant for Crohn disease in atg16/1. Nat. Genet. 39, 207-211 (2007).

6. Cadwell, K. et al. A key role for autophagy and the autophagy gene atg16l1 in mouse and human intestinal paneth cells. Nature 456, 259-263 (2008).

7. Sadaghian Sadabad, M. et al. The atg16l1-t300a allele impairs clearance of pathosymbionts in the inflamed ileal mucosa of Crohn's disease patients. Gut 64, 1546-1552 (2015).

8. Adolph, T.E. et al. Paneth cells as a site of origin for intestinal inflammation. Nature 503, 272-276 (2013).

9. Birmingham, C.L., Smith, A.C., Bakowski, M.A., Yoshimori, T. \& Brumell, J.H. Autophagy controls salmonella infection in response to damage to the salmonella-containing vacuole. J. Biol. Chem. 281, 11374-11383 (2006).

10. Gutierrez, M.G. et al. Autophagy is a defense mechanism inhibiting bcg and mycobacterium tuberculosis survival in infected macrophages. Cell 119, 753-766 (2004).

11. Schmid, D., Pypaert, M. \& Munz, C. Antigen-loading compartments for major histocompatibility complex class ii molecules continuously receive input from autophagosomes. Immunity 26, 79-92 (2007).

12. Saitoh, T. et al. Loss of the autophagy protein atg16l1 enhances endotoxininduced il-1beta production. Nature 456, 264-268 (2008).

13. Wildenberg, M.E. et al. Autophagy attenuates the adaptive immune response by destabilizing the immunologic synapse. Gastroenterology 142, 1493-1503 (2012).

14. Poppe, D. et al. Azathioprine suppresses ezrin-radixin-moesin-dependent t cell-apc conjugation through inhibition of vav guanosine exchange activity on rac proteins. J. Immunol. 176, 640-651 (2006).

15. Tiede, I. et al. Cd28-dependent rac1 activation is the molecular target of azathioprine in primary human cd4 $+\mathrm{t}$ lymphocytes. J. Clin. Invest. 111, 1133-1145 (2003).

16. Hall, A. Rho gtpases and the actin cytoskeleton. Science $279,509-514$ (1998).

17. Ridley, A.J. et al. Cell migration: integrating signals from front to back. Science 302, 1704-1709 (2003).

18. Pagler, T.A. et al. Deletion of abca1 and abcg1 impairs macrophage migration because of increased rac1 signaling. Circ. Res. 108, 194-200 (2011).
19. Wheeler, A.P. et al. Rac1 and rac2 regulate macrophage morphology but are not essential for migration. J. Cell Sci. 119, 2749-2757 (2006).

20. Park, Y.M. et al. Oxidized ldl/cd36 interaction induces loss of cell polarity and inhibits macrophage locomotion. Mol. Biol. Cell 23, 3057-3068 (2012).

21. Colombel, J.F. et al. Infliximab, azathioprine, or combination therapy for Crohn's disease. N. Engl. J. Med. 362, 1383-1395 (2010).

22. Thrasher, A.J. \& Burns, S.O. Wasp: a key immunological multitasker. Nat. Rev. Immunol. 10, 182-192 (2010).

23. Nguyen, D.D. et al. Wiskott-Aldrich syndrome protein deficiency in innate immune cells leads to mucosal immune dysregulation and colitis in mice. Gastroenterology 143, 719-729 (2012).

24. Carroll, B. et al. The tbc/rabgap armus coordinates rac1 and rab7 functions during autophagy. Dev. Cell 25, 15-28 (2013).

25. Nethe, M. et al. Focal-adhesion targeting links caveolin-1 to a rac1degradation pathway. J. Cell Sci. 123, 1948-1958 (2010).

26. Kim, P.K., Hailey, D.W., Mullen, R.T. \& Lippincott-Schwartz, J. Ubiquitin signals autophagic degradation of cytosolic proteins and peroxisomes. Proc. Natl. Acad. Sci. USA 105, $20567-20574$ (2008).

27. Pertz, O. Spatio-temporal rho gtpase signaling-where are we now? J. Cell Sci. 123, 1841-1850 (2010).

28. Ravindran, R. et al. The amino acid sensor gen 2 controls gut inflammation by inhibiting inflammasome activation. Nature 531, 523-527 (2016).

29. D'Alessio, S. et al. Vegf-c-dependent stimulation of lymphatic function ameliorates experimental inflammatory bowel disease. J. Clin. Invest. 124, 3863-3878 (2014).

30. du Pre, M.F. \& Samsom, J.N. Adaptive t-cell responses regulating oral tolerance to protein antigen. Allergy 66, 478-490 (2011).

31. Spahn, T.W. et al. Mesenteric lymph nodes are critical for the induction of high-dose oral tolerance in the absence of Peyer's patches. Eur. J. Immunol. 32, 1109-1113 (2002).

32. Parikh, K. et al. Suppression of p21rac signaling and increased innate immunity mediate remission in Crohn's disease. Sci. transl. med. 6, 233ra253 (2014).

33. Hara, T. et al. Suppression of basal autophagy in neural cells causes neurodegenerative disease in mice. Nature 441, 885-889 (2006).

34. Johnsson, A.K. et al. The rac-fret mouse reveals tight spatiotemporal control of rac activity in primary cells and tissues. Cell Rep. 6, 1153-1164 (2014).

35. Nobis, M. et al. Intravital flim-fret imaging reveals dasatinib-induced spatial control of src in pancreatic cancer. Cancer Res. 73, 4674-4686 (2013).

36. Weersma, R.K. et al. Confirmation of multiple crohn's disease susceptibility loci in a large dutch-belgian cohort. Am. J. Gastroenterol. 104, 630-638 (2009).

37. Wolfkamp, S.C. et al. Atg16l1 and nod2 polymorphisms enhance phagocytosis in monocytes of Crohn's disease patients. World J. Gastroenterol. 20, 2664-2672 (2014). 